\title{
Sevelamer carbonate in the treatment of hyperphosphatemia in patients with chronic kidney disease on hemodialysis
}

\author{
Vincenzo Savica ${ }^{1,2}$ \\ Domenico Santoro' \\ Paolo Monardo 2 \\ Agostino Mallamace' \\ Guido Bellinghieri' \\ 'Experimental and Clinic \\ Department of Internal Medicine \\ and Pharmacology, University \\ of Messina, Italy; ${ }^{2} \mathrm{Nephrology}$ \\ and Dialysis Unit, Papardo Hospital, \\ Messina, Italy
}

\begin{abstract}
Sevelamer carbonate is an anion exchange pharmaceutical, developed to improve on the performance of the non-absorbable, non-calcium, and metal-free phosphate binder sevelamer hydrochloride. Sevelamer carbonate is expected not to worsen metabolic acidosis, as previously reported during long-term treatment with sevelamer hydrochloride in hemodialysis (HD) patients. Carbonate is the alternate counterion to chloride on the sevelamer polymeric backbone, but the active poly(allylamine) responsible for phosphate $\left(\mathrm{PO}_{4}\right)$ binding remains unaltered. Therefore, sevelamer carbonate is expected to reduce elevated serum phosphorus level, similarly to sevelamer hydrochloride. Sevelamers are prescribed in uremic HD patients to control hyperphosphatemia, but the carbonate has also been proposed for the treatment of chronic kidney disease (CKD) non-dialysis patients. Although hyperphosphatemia is regarded as a main contributor to increased mortality in the HD population because of cardiovascular calcification, metabolic acidosis has also been advocated as a major player in the increased mortality in this population, by engendering malnutrition, negative nitrogen balance, and inflammation. This paper reviews the evidence showing that sevelamer carbonate is as good as sevelamer hydrochloride in terms of hyperphosphatemia control in CKD, but with a better outcome in serum bicarbonate balance.
\end{abstract}

Keywords: chronic kidney disease, sevelamer carbonate, hyperphosphatemia, hemodialysis

\section{Introduction}

Chronic kidney disease (CKD) has become a major worldwide healthcare problem, affecting an estimated $5 \%-10 \%$ of the world's population (Hamer and El Nahas 2006). Progression to end stage renal disease (ERSD), the need for renal replacement therapy, and the high annual death rate of dialysis patients are the most noticeable outcomes of CKD. Most patients with CKD in fact die mainly from cardiovascular disease, rather than from ERSD. Coronary artery calcification (CAC), a surrogate marker of atherosclerosis, is a common finding in CKD. Extensive calcification has been documented in dialysis patients by computed tomography, but cardiovascular calcification (CVC) affects patients not undergoing dialysis as well, developing early in the progression of CKD, and progressively worsening with the decline of the glomerular filtration rate (GFR), particularly in diabetics progressing to ERSD (Qunibi 2007). Presence of proteinuria, reduced renal function, diabetic nephropathy, and the progression rate to ERSD are the classical main uremia-related factors that increase the risk of calcification in CKD. Several observational studies have now identified the altered mineral metabolism, and particularly hyperphosphatemia, as a key player in CVC and not exclusively in musculoskeletal health. Hyperphosphatemia associated with surrogate clinical events such as CAC, aortic calcification, valvular calcification, aortic stiffness, pulse pressure, as well as with hard clinical outcomes such as 
hospitalization, and all-cause and cardiovascular mortality (Young et al 2005; Young 2007; Toussaint and Kerr 2007). Elevated serum phosphorus (P) level is highly prevalent in uremic patients, despite diet restriction and dialysis. It is associated with an increased mortality risk in hemodialysis (HD) patients. Block et al (1998), through a multivariate analysis of data from the United States Renal Data System (URDS), identified elevated serum $P$ level as an independent predictor of mortality. The overall mortality risk associated with serum $P$ above $6.5 \mathrm{mg} / \mathrm{dL}$ was $27 \%$ greater than that of patients with serum P between 2.4 and $6.5 \mathrm{mg} / \mathrm{dL}$. Moreover, $\mathrm{Ca} \times \mathrm{P}$ product greater than $72 \mathrm{mg}^{2} / \mathrm{dL}^{2}$ was also associated with increased mortality risk. Altered mineral metabolism could aggravate the effects of coronary atherosclerosis. An increased intracellular P intake via Pit-1, a sodium-dependent $\mathrm{P}$ co-transporter, stimulates the phenotypic conversion of smooth muscle cell to osteoblatic cell lineage, thus leading to an increased extracellular matrix deposition favoring $\mathrm{Ca} \times \mathrm{P}$ product precipitation, with the proposed final outcome of vascular stiffness (Giachelli 2003; Li et al 2006). In primary cultures of vascular smooth muscle cells derived from atherosclerotic human aortas, activation of osteoblast specific transcriptional programs related to skeletal morphogenesis did not lead to matrix mineralization until the $\mathrm{P}$ concentration of the tunica media was increased, an event occurring only after the onset of renal dysfunction and hyperphosphatemia (Mathew et al 2008). Indeed, using high-resolution B-mode ultrasonography, Kawagishi et al (1995) found that elevated serum $P$ level was strongly associated with changes in intimamedia thickness of the carotid artery, an effect independent of several other commonly measured coronary risk factors. It was therefore hypothesized that the increased mortality risk associated with elevated $P$ level in HD patients was primarily due to cardiac rather than non-cardiac causes of death. In a chronic HD patients with serum $\mathrm{P}>6.5 \mathrm{mg} / \mathrm{dL}$, Ganesh et al (2001) demonstrated a $41 \%$ greater risk of death resulting from coronary artery disease (CAD) and a 20\% greater risk of death resulting from sudden death compared with patients with serum P level between 2.4 and $6.5 \mathrm{mg} / \mathrm{dL}$. Comparing death risk between CAD and non-CAD causes of death in HD patients, he showed that elevated serum $P$ level in HD patients significantly and preferentially predisposes to CAD deaths, thus supporting the concept of hyperphosphatemia as a cardiotoxin. The author suggested a role for elevated serum $\mathrm{P}$ either in the development, progression, or rupture of atheromatous plaques in the coronary arteries of prevalent ERSD patients. All the previous analysis involved HD populations with serum $\mathrm{P}>6.5 \mathrm{mg} / \mathrm{dL}$, which means a
$\mathrm{P}$ value at least $1 \mathrm{mg} / \mathrm{dL}$ higher than the National Kidney Foundation (K/DOQI) recommended upper limit of $5.5 \mathrm{mg} /$ $\mathrm{dL}$ for chronic HD patients. Interestingly, both in CKD patients with serum $\mathrm{P}$ within the $\mathrm{K} / \mathrm{DOQI}$ recommended serum $P$ level range $(>2.7$ and $<4.5 \mathrm{mg} / \mathrm{dL})$, as in the case of stage 3 CKD patients (Kestenbaum et al 2005), and in stage 4 CKD patients with elevated serum $P$ but not yet in dialysis, arterial stiffness and mortality risk from cardiovascular events are significantly increased (Sigrist et al 2007).

\section{Hyperphosphatemia and phosphate binders}

The evidence that links mortality with altered mineral metabolism, and particularly the association of mortality with elevated serum $\mathrm{P}$ and $\mathrm{Ca} \times \mathrm{P}$ levels, is well documented. Therefore, mineral metabolism provides a new perspective for improving mortality in patients with kidney disease. Hyperphosphatemia requires strict management through dietary restriction, dialysis, and use of phosphate binders. Phosphate binder therapy is a critical factor in the management of hyperphosphatemia in advanced kidney disease. The aim is to achieve serum $\mathrm{P} \leq 5.5 \mathrm{mg} / \mathrm{dL}$ in HD patients, and possibly even lower. Phosphate binders mainly act in reducing the amount of bioavailable $\mathrm{PO}_{4}$ generated by food intake into gastrointestinal fluids, through precipitation and/or entrapment, and potentiating its excretion by the fecal route. Several options are commercially available, namely: calcium carbonate and calcium acetate, magnesium hydroxide and magnesium carbonate, aluminium hydroxide, lanthanum carbonate, the non-calcium and metal-free sevelamer hydrochloride (for review: Almirall and Valenzuela 2006; Cozzolino et al 2008), and sevelamer carbonate (Renvela ${ }^{\circledR}$ which obtained FDA approval in October 2007, but is not approved yet in EU). Others substances are currently under development (McIntyre 2007). It is beyond the aim of this review to provide details on all the above-listed phosphate binders. Nevertheless, it is worth noting that each of those listed, although to various degrees effective in serum $P$ reduction, is not devoid of concern (for review: Almirall and Valenzuela 2006). Systemic toxicity, observed as liver, lung, and kidney deposition, and neurotoxicity, has been reported for lanthanum carbonate at the experimental level (Lacour et al 2005, 2007; Slatopolsky et al 2005), but not yet in the clinical setting (Cozzolino et al 2008), and some criticism remains because of past experience with another metal-based P binder, aluminium hydroxide (Drüeke 2007). An increase in vascular calcification, suggestive of a possibly increased mortality risk, has been advocated for calcium-based 
$\mathrm{PO}_{4}$ binders. Intolerance to sevelamer in $9 \%-34 \%$ of $\mathrm{HD}$ patients because of gastrointestinal complaints (Almirall and Valenzuela 2006), worsening of metabolic acidosis (De Santo et al 2006), reports of stercoral ulceration (Madan et al 2008), and peritonitis (EMEA 2007) have been highlighted for sevelamer hydrochloride. The Dialysis Outcomes and Practice Patterns Study (DOPPS) revealed considerable variation in the use of phosphate binders among the seven different countries participating in the study, although calcium-based binders were the preferred option (Young et al 2005). At that time, the use of non-calcium and metal-free polymers such as sevelamer was clearly underestimated because of the very recent approval of the pharmaceutical. Nowadays, unexpectedly, most patients are still prescribed calcium-based agents, despite evidence of increased vascular calcification, arterial stiffness, and coronary calcification both in HD and predialysis patients (Block et al 2007; Russo et al 2007; Sigrist et al 2007). The widespread use of calcium-based binders could have several explanations. Some authors have reported that calcium-based salts in HD patients showed good phosphatebinding capability, cost affordability, and lack of compelling evidence for a significantly reduced outcome in overall mortality compared with sevelamer (St. Peter et al 2008; Suki 2008). Others, however, have interpreted the reduced calcification in sevelamer-treated patients by its lipid-lowering properties more than its phosphate-binding properties (Winkelmayer and Tonelli 2008).

\section{Sevelamer carbonate in HD patients}

Sevelamer carbonate is an anion exchange pharmaceutical, recently developed as an improvement of the non-absorbable sevelamer hydrochloride. It was developed to avoid worsening of metabolic acidosis, reported as one of the possible concerning side effects of sevelamer hydrochloride long-term treatment, and to increase patients' compliance through the development of new pharmaceutical presentation (Duggal et al 2006). Sevelamer hydrochloride is a non-calcium and non-metal quaternary amine anion exchange resin, which upon binding to a $\mathrm{PO}_{4}$ molecule by ionic and/or hydrogen bonding releases one hydrochloride molecule. For this reason, sevelamer hydrochloride has been demonstrated to worsen the metabolic acidosis that affects HD patients, leading to a serum bicarbonate threshold of $17-19 \mathrm{mEq} / \mathrm{L}$, below the recommended K/DOQI level of $22 \mathrm{mEq} / \mathrm{L}$ (De Santo et al 2006). There are abundant data from short-term metabolic studies in patients with CKD indicating that metabolic acidosis, a common condition in renal insuffi- ciency, may set up or worsen protein-energy malnutrition, inflammation, and bone disease. Although carbonate is the alternate counterion to chloride on the polymer backbone of sevelamer, the active poly(allylamine) responsible for phosphate $\left(\mathrm{PO}_{4}\right)$ binding remains unaltered between the two sevelamers. Therefore, it could be expected that sevelamer carbonate is acting similarly to sevelamer hydrochloride in serum $\mathrm{P}$ and lipid reduction, due to the fact that the anion does not play any role in phosphate binding. Together with dietary restriction and dialysis, sevelamer is currently prescribed in uremic HD patients to control hyperphosphatemia. Sevelamer, either hydrochloride or carbonate, exerts its action via the reduction of the amount of bioavailable P generated from food intake and intestinally absorbed from gastrointestinal fluids. In healthy patients, a poorly regulated $\mathrm{P}$ intestinal absorption is balanced by an efficient urinary $\mathrm{P}$ excretion, thus warranting normophosphatemia, but when advanced renal failure develops, an inefficient or absent $\mathrm{P}$ excretion no longer balances the unrelenting intestinal P absorption, thus leading to hyperphosphatemia. Looking at the clinical evidence produced with sevelamer carbonate to date, sevelamer carbonate has been demonstrated to be as effective as sevelamer hydrochloride in terms of serum $P$ and serum lipid reduction in two different studies, one involving ESRD patients in hemodialysis and the other involving $\mathrm{CKD}$, non-dialysis patients. A randomized, double blind, crossover design study was performed in HD patients to document the substantial equivalence of sevelamer carbonate with the hydrochloride form (Delmez et al 2007). Patients were eligible if they were on HD 3 times per week for at least 3 months. Ninety-two percent of the enrolled population was maintained on sevelamer hydrochloride as the sole phosphate binder at a total daily dose of $\leq 13.6 \mathrm{~g}$, with or without vitamin D analog and/or lipid lowering therapy, and patients were reported to be historically well controlled in terms of serum P $(3.5-6.5 \mathrm{mg} / \mathrm{dL})$. After a 5 -week run-in period to titrate the dose of sevelamer hydrochloride, cinacalcet, and vitamin D analog, $79 \mathrm{HD}$ patients were randomly assigned in a 1:1 fashion to either sevelamer carbonate or sevelamer hydrochloride for 8 weeks followed by a crossover to the other treatment for additional 8 weeks. Sevelamer starting dose was individualized to the prescribed dose during the run-in period. Three subgroups of patients could be roughly identified on the basis of the daily dosage: (a) $\leq 4.8$, (b) $>4.8$ to 9.6 , and (c) $\geq 9.6 \mathrm{~g} /$ day. Unfortunately, the paper by Delmez et al (2007) did not report the individual serum P level, total cholesterol, LDL, and serum bicarbonate, or the average serum P level of the three subgrouped patients 
according to the need of a so strikingly different daily dosing, determined at the end of the 5-week run-in period. Patients were instructed to maintain the same daily dose throughout the full observational period, ie, 16 weeks. In the equivalence testing with a $90 \% \mathrm{CI}$ for $\log$-transformed serum $\mathrm{P}$ within the range of $0.80-1.25$, the mean serum $P$ was $4.6 \pm 0.9 \mathrm{mg} / \mathrm{dL}$ during sevelamer carbonate treatment and $4.7 \pm 0.9 \mathrm{mg} / \mathrm{dL}$ during sevelamer hydrochloride treatment. Efficacy assessment was run in the per protocol set (PPS), thus excluding protocol deviations expected to bias the results. Seventy-one percent of patients during sevelamer carbonate and $70 \%$ during sevelamer hydrochloride treatment were within the recommended serum $P$ range. At the end of each treatment, $41 \%$ of patients treated with sevelamer carbonate and $53 \%$ of patients treated with sevelamer hydrochloride had a serum bicarbonate level lower than the recommended value of 22 $\mathrm{mEq} / \mathrm{L}$. These results suggest a better outcome in serum bicarbonate at the end of sevelamer carbonate treatment, whereas patients during sevelamer hydrochloride treatment presented with lower serum values of total cholesterol and LDL cholesterol, suggestive of a better outcome in lipid lowering profile at the end of sevelamer hydrochloride treatment compared with the carbonate.

It has to be mentioned that recently Qunibi et al (2008) showed that the reduction of low-density lipoprotein cholesterol (LDL-C) levels may be responsible for the lowering of CAC in patients treated with sevelamer hydrochloride. Indeed, they showed that after intensive lowering of LDL-C levels for 1 year with atorvastatin, hemodialysis patients treated with either calcium acetate or sevelamer experienced similar progression of CAC (Qunibi et al 2008).

\section{Sevelamer carbonate in hyperphosphatemic, non-dialysis patients}

In a recent open label, single arm study, Ketteler et al (2008) administered sevelamer carbonate at a mean actual daily dosage of $5.5 \mathrm{~g}$ (6-7 sevelamer carbonate $800 \mathrm{mg}$ tablets per day) to $C K D$ patients with mean serum $P \geq 6.2 \pm 0.8$ $\mathrm{mg} / \mathrm{dL}$ and not under replacement therapy. A significant 1.4 $\mathrm{mg} / \mathrm{dL}$ decrease in serum $\mathrm{P}$ was observed over an 8-week treatment, after a 2-week washout for patients previously on other phosphate binders, mainly calcium-based phosphate binders. Full recovery of serum $\mathrm{P}$ at baseline level was reached after the 2 -week post-treatment washout period. By the end of sevelamer carbonate treatment, $75 \%$ of stage 4 CKD patients (GFR 15-29 mL/min per $1.73 \mathrm{~m}^{2} ; 35 \%$ of study population) fell into the $\mathrm{K} / \mathrm{DOQI}$ recommended $\geq 2.7$ and $\leq 4.6 \mathrm{mg} / \mathrm{dL}$ serum $\mathrm{P}$ range, and $70 \%$ of stage $5 \mathrm{CKD}$ patients (GFR $<15 \mathrm{~mL} / \mathrm{min}$ per $1.73 \mathrm{~m}^{2} ; 65 \%$ of study population) had achieved a serum $\mathrm{P}$ level $\leq 5.5 \mathrm{mg} / \mathrm{dL}$. A statistically significant decrease in serum $\mathrm{Ca} \times \mathrm{P}$ product, total cholesterol, and serum LDL, and notably an increase in serum $\mathrm{Ca}$ and serum bicarbonate were also observed at the end of treatment.

\section{Discussion}

While exerting a lower control of hyperphosphatemia, sevelamer hydrochloride was demonstrated to worsen the metabolic acidosis compared with calcium carbonate, significantly decreasing the plasma bicarbonate concentrations to the threshold of $17 \mathrm{mEq} / \mathrm{L}$ and reducing the serum albumin levels over a 24-week treatment in HD patients (De Santo et al 2006). Metabolic acidosis has been reported to act as a uremic toxin, and the recommended K/DOQI predialysis bicarbonate concentration is $22 \mathrm{mEq} / \mathrm{L}$. Forty-one percent of HD patients during sevelamer carbonate treatment, and $53 \%$ during sevelamer hydrochloride treatment, were below the recommended K/DOQI threshold. The increase in serum bicarbonate observed with sevelamer carbonate may therefore confer an increased benefit over sevelamer hydrochloride treatment, which is acid-base neutral or potentially acidifying.

Acidosis has been associated with adverse effects on bone metabolism (Kraut 1995) and increased malnutrition and inflammation (Kalantar-Zadeh et al 2004) in HD patients. Oral bicarbonate supplementation was found to result in fewer hospital admissions and fewer days hospitalized in peritoneal dialysis patients (Szeto et al 2003). Acidosis is commonly seen in patients with CKD, and these effects may be of more concern in the predialysis patients, for whom the dialysis modality itself is not being used to correct these abnormalities. For this reason, sevelamer carbonate's manufacturer is proposing the product as preferable option in CKD non-dialysis patients for phosphatebinding therapy.

The need for phosphate-binding therapy in uremic patients is a cornerstone in patient management. When renal failure occurs and insignificant $P$ renal excretion does not balance the unrelenting absorption of phosphate from gastrointestinal fluids, the reduction of the amount of bioavailable phosphate generated by dietary intake remains the only way to reduce hyperphosphatemia. Sevelamers seem to present some advantages over other still largely prescribed phosphate binders, such as aluminium hydroxide and calcium-based binders, because of no systemic toxicity on the one hand and no additional calcium load on the other, this latter condition 
being particularly relevant when considering recent evidence of increased vascular calcification by calcium-based binders. Even if secondary analysis of the DCOR study showed that treatment with sevelamer compared with calcium-based binders was not correlated with a reduction of overall mortality, cause-specific mortality, morbidity, or first or cause-specific hospitalization, the authors observed a beneficial effect on multiple all-cause hospitalizations and hospital days, which are important because cost-analysis represents a major problem in treating dialysis patients.

Nevertheless, as can be inferred from the Delmez et al (2007) study on sevelamer carbonate in HD patients, compliance with a daily dosage requiring up to $13.6 \mathrm{~g}$ remains a major problem, either for pharmacoeconomic affordability and for adherence to the multi-approach therapy, commonly prescribed to dialysis patients requiring a relevant daily pill burden. The better outcome of sevelamer carbonate on serum bicarbonate balance over a bioequivalent reduction of hyperphosphatemia compared with sevelamer hydrochloride is noteworthy. However, only approximately $50 \%$ of dialysis patients under sevelamer hydrochloride treatment achieve the recommended $\mathrm{K} / \mathrm{DOQI}$ serum P level of $5.5 \mathrm{mg} / \mathrm{dL}$. Based on its bioequivalence to hydrochloride in the reduction of hyperphosphatemia, the same percentage of treatment success could be expected for sevelamer carbonate. Therefore, although nephrologists have several choices in phosphate-binding therapy, currently no gold standard seems to exist or to be preferably recommendable among commercially available competitors.

\section{Disclosures}

None of the authors has any conflicts of interest to declare.

\section{References}

Almirall Daly J, Valenzuela MP. 2006. The safety of phosphate binders. Expert Opin Drug Saf, 5:675-86.

Block GA, Hulbert-Shearon TE, Levin NW, et al. 1998. Association of serum phosphorus and calcium $\times$ phosphate product with mortality risk in chronic hemodialysis patients: a national study. Am J Kidney Dis, 31:607-17.

Block GA, Raggi P, Bellasi A, et al. 2007. Mortality effect of coronary calcification and phosphate binder choice in incident hemodialysis patients. Kidney Int, 71:438-41.

Cozzolino M, Galassi A, Pasho S, et al. 2008. Preventive measures and new pharmacological approaches of calcium and phosphate disorders. Contrib Nephrol, 161:234-9.

De Santo NG, Frangiosa A, Anastasio P, et al. 2006. Sevelamer worsens metabolic acidosis in hemodialysis patients. J Nephrol, 19(S9):S108-14.

Delmez J, Block G, Robertson J, et al. 2007. A randomized, double-blind, crossover design study of sevelamer hydrochloride and sevelamer carbonate in patients on hemodialysis. Clin Nephrol, 68:386-91.

Drüeke TB, 2007. Lanthanum carbonate as a first-line phosphate binder: the "cons". Semin Dial, 20:329-32.

Duggal A,Hanus M, Zhorov E, et al. 2006. Novel dosage forms and regimens for sevelamer-based phosphate binders. J Ren Nutr, 16:248-52.
EMEA. 2007. Scientific discussion on Renagel. Procedure EMEA/H/ C000254/II/56, 1 June 2007.

Ganesh SK, Stack AG, Levin NW et al. 2001. Association of elevated serum $\mathrm{PO} 4, \mathrm{Ca} \times \mathrm{PO} 4$ product, and paratyroid hormone with cardiac mortality risk in chronic hemodialysis patients. J Am Soc Nephrol, 12:2131-8.

Giachelli CM. 2003. Vascular calcification: in vitro evidence for the role of inorganic phosphate. J Am Soc Nephrol 14:S300-4.

Hamer RA, El Nahas M. 2006. The burden of chronic kidney disease. $B M J, 332: 563-4$.

Kalantar-Zadeh K, Mehrotra R, Fouque D, et al. 2004. Metabolic acidosis and malnutrition-inflammation complex syndrome in chronic renal failure. Semin Dial, 17:455-65.

Kawagishi T, Nishizawa Y, Konishi T, et al. 1995. High-resolution B-mode ultrasonography in evaluation of atherosclerosis in uremia. Kidney Int, 48:820-6.

Kestenbaum B, Sampson JN, Rudser KD, et al. 2005. Serum phosphate levels and mortality risk among people with chronic kidney disease. $J$ Am Soc Nephrol, 16:520-8.

Ketteler M, Rix M, Fan S, et al. 2008. Efficacy and tolerability of sevelamer carbonate in hyperphosphatemic patients who have chronic kidney disease and are not on dialysis. Clin J Am Soc Nephrol, doi 10.2215/ CJN.05161107.

Kraut JA. 1995. The role of metabolic acidosis in the pathogenesis of renal osteodystrophy. Adv Ren Replace Ther, 2:40-51.

Lacour B, Lucas A, Auchere D, et al. 2005. Chronic renal failure is associated with increase tissue deposition of lanthanum after 28-day oral administration. Kidney Int, 67:1062-69.

Lacour B, Nikolov IG, Joki N. 2007. Lanthanum carbonate, body lanthanum accumulation and potential liver toxicity. Nephrol Dial Transplant, 22:1788-99.

Li X, Yang HY, Giachelli CM. 2006. Role of sodium-dependent phosphate co-transporter, Pit-1, in vascular smooth cell calcification. Circ Res, 98:905-12.

Madan P, Bhayana S, Chandra P, et al. 2008. Lower gastrointestinal bleeding: association with sevelamer use. World J Gastroenterol, 14:2615-6.

Mathew S, Tustison KS, Sugatani T, et al. 2008. The mechanism of phopshorus as a cardiovascular risk factor in CKD. JASN, doi:10.1681/ ASN.20070707060.

McIntyre CW. 2007. New developments in the management of hyperphosphatemia in chronic kidney disease. Semin Dial, 20:337-41.

Qunibi WY. 2007. Cardiovascular calcification in nondialyzed patients with chronic kidney disease. Semin Dial, 20:134-8

Qunibi W, Moustafa M, Muenz LR, et al. CARE-2 Investigators. 2008. A 1-year randomized trial of calcium acetate versus sevelamer on progression of coronary artery calcification in hemodialysis patients with comparable lipid control: The Calcium Acetate Renagel Evaluation-2 (CARE-2) Study. Am J Kidney Dis, 51:952-65.

Russo D, Miranda I, Ruocco C, et al. 2007. The progression of coronary artery calcification in predialysis patients on calcium carbonate or sevelamer. Kidney Int, 72:1255-61.

Sigrist MK, Taal MW, Bungay P, et al. 2007. Progressive vascular calcification over 2 years is associated with arterial stiffening and increased mortality in patients with stages 4 and 5 chronic kidney disease. Clin $J$ Am Soc Nephrol, 2:1241-8.

St. Peter WL, Liu J, Weinhandl E, et al. 2008. A comparison of sevelamer and calcium-based phosphate binders on mortality,hospitalization, and morbidity in hemodialysis: a secondary analysis of the dialysis clinical outcomes revisited (DCOR) randomized trial using claims data. Am J Kidney Dis, 51:445-54.

Suki WN. 2008. Effects of sevelamer and calcium-based phosphate binders on mortality in hemodialysis patients: results of a randomized clinical trial. J Ren Nutr, 18:91-8.

Szeto CC, Wong TY, Chow KM, et al. 2003. Oral sodium bicarbonate for the treatment of metabolic acidosis in peritoneal dialys patients: a randomized placebo-control trial. J Am Soc Nephrol, 14:2119-26. 
Toussaint ND, Kerr PG. 2007. Vascular calcification and arterial stiffness in chronic kidney disease: implications and management. Nephrology, 12:500-9.

Winkelmayer WC, Tonelli M. 2008. Phosphate binder choice in dialysis patients: a call for evidence.based rather than marketing-based clinical practice. Am J Kidney Dis, 51:362-5.
Young EW, Albert JM, Satayathum S e al. 2005. Predictors and consequences of altered mineral metabolism: the dialysis outcomes and practice patterns study. Kidney Int, 67:1179-87.

Young EW. 2007. Mineral metabolism and mortality in patients with chronic kidney disease. Adv Chronic Kidney Dis, 14:13-21. 